\title{
A randomised trial on the therapeutic effectiveness of bronchoalveolar lavage under fiberoptic bronchoscopy in patients with severe lung infection living in the Tibetan plateau area
}

\author{
Wugang Zhou ${ }^{1 "}$, Chi Zhou ${ }^{2 \#}$, Xuqin Liu ${ }^{3}$, Ningning $\mathrm{Shi}^{3}$, Wangmu Quyang ${ }^{3}$, Dan $\mathrm{Tu}^{3}$, Yong $\mathrm{Xin}^{3}, \mathrm{Lv} \mathrm{Ji}^{3}$ \\ ${ }^{1}$ Department of Emergency, Shanghai Ninth People's Hospital, School of Medicine, Shanghai Jiao Tong University, Shanghai, China; ${ }^{2}$ Department \\ of Anesthesiology, Shanghai Ninth People's Hospital, School of Medicine, Shanghai Jiao Tong University, Shanghai, China; ${ }^{3}$ Department of Intensive \\ Care Unit, Shigatse People's Hospital, Shigatse, Tibet Autonomous Region, China \\ Contributions: (I) Conception and design: W Zhou, L Ji; (II) Administrative support: W Zhou; (III) Provision of study materials or patients: W Zhou, \\ L Ji; (IV) Collection and assembly of data: All authors; (V) Data analysis and interpretation: W Zhou, C Zhou, L Ji; (VI) Manuscript writing: All \\ authors; (VII) Final approval of manuscript: All authors. \\ \#These authors contributed equally to this work. \\ Correspondence to: Lv Ji. Department of Intensive Care Unit, Shigatse People's Hospital, No. 1, Daqing East Road, Shigatse 857000, Tibet \\ Autonomous Region, China. Email: jilv_1907@163.com.
}

Backgroundk People living in plateau areas are prone to upper respiratory tract infections and secondary lung infections. The current study aimed to explore the effects of bronchoalveolar lavage under fiberoptic bronchoscope for the treatment of patients with severe pulmonary infection living in plateau areas.

Methods: 148 patients with severe lung infection admitted to the intensive care unit of Shigatse People's Hospital (Shigatse, Tibet Autonomous Region, China) between July 2019 and January 2021 were enrolled. Patients were randomly assigned to the observational group or the control group. For all patients, basic clinical data including sex, age, body mass index (BMI), blood pressure, diabetes history, stroke history, presence or absence of chronic obstructive pulmonary disease, lung infection (gram-positive bacterial infection, gram-negative bacterial infection, fungal infection, acute lung abscess), surgical history, and postoperative inhalation injury. were collected. The control group received conventional treatment, and the observational group received bronchoalveolar lavage under fiberoptic bronchoscopy. Pearson's correlation was used to analyze the correlations between bronchoalveolar lavage under fiberoptic bronchoscopy and inflammatory factors levels. Logistic regression was used to investigate the correlation between bronchoalveolar lavage under fiberoptic bronchoscopy and the effectiveness of the treatment.

Results: Before treatment, no significant difference existed in the basic data of the observational group and the control group. After treatment, the parameters of respiratory mechanics and inflammatory factors in the 2 groups were significantly improved compared with those before treatment $(\mathrm{P}<0.05)$. At the same time, in the observational group, the clinical parameters were significantly higher than those of the control group, and the levels of inflammatory factors were significantly lower than those of the control group (all $\mathrm{P}<0.05$ ). After full adjustment for age, sex, BMI, gram-negative infection, diabetes, and acute lung abscess, compared with the control group, the therapeutic effectiveness in the observational group was increased by $23 \%$ (OR $=1.23,95 \%$ CI: $1.09-1.51, \mathrm{P}=0.007)$.

Conclusions: For patients with severe lung infection who are resident in high altitude areas, compared with conventional treatments, bronchoalveolar lavage under fiberoptic bronchoscopy can significantly improve chest, lung, and total dynamic compliance, as well as reduce the levels of the inflammatory factors procalcitonin (PCT) and transforming growth factor- $\beta$, thus increasing the effectiveness of the treatment.

Keywords: Bronchoscopy; severe pulmonary infection; plateau area 
Submitted Jan 27, 2021. Accepted for publication Mar 16, 2021.

doi: 10.21037/apm-21-470

View this article at: http://dx.doi.org/10.21037/apm-21-470

\section{Introduction}

Pulmonary infection is characterized by substantial inflammation of the alveolar cavity, the interstitium of the lungs, and terminal airways. It is mainly caused by virulent bacterial infection, including by drug-resistant bacteria, or infection with multiple bacteria simultaneously, with Staphylococcus aureus and Streptococcus pneumoniae being the most frequent culprit pathogens. The major clinical characteristics of pulmonary infection include cough, increased respiratory secretions, and weakness $(1,2)$. The severe pulmonary infection is a serious lung illness accompanied by varying degrees of abnormal parameters of respiratory mechanics which can stimulate the overexpression of multiple inflammatory factors. Among these overexpressed inflammatory factors, procalcitonin (PCT) and transforming growth factor $\beta$ (TGF- $\beta$ ) are the most significant. Therefore, measurement of the levels of these 2 growth factors can be used to evaluate the severity of pulmonary infection $(3,4)$.

Year-round residents of plateau areas are prone to developing upper respiratory tract infections and secondary pulmonary infections. Long-term exposure to low oxygen, low air pressure, and cold can cause dryness and congestion of the upper respiratory tract, which is conducive to respiratory infections. If a severe pulmonary infection is complicated by a serious condition, a delay in treatment may even result in acute respiratory failure, which dramatically threatens the health and life of the patient $(5,6)$. Timely control of the infected lesions is important for the effective treatment. At present, anti-infective treatment is still in a simple and systematic research stage. The effective concentration of drugs in the local lesions is often low; moreover, the application of broad-spectrum antibacterial drugs has led to an increase in drug-resistant strains, which makes constraining infections even more difficult to effectively accomplish (7). The key to treating a severe pulmonary infection is to support the patient to breathe smoothly so that the medicine can work more effectively. Increased lung secretions have been reported to lead to airway obstruction (8), increase the difficulty of expectoration, induce the accretion of irritating substances, and induce a series of cascade reactions. As a precision inspection method, fiberoptic bronchoscopy is widely used to diagnose and treat a variety of bronchial illnesses. It can eliminate sputum and visible inflammatory secretions, and can help to completely flush the patient's lesions, therefore allowing for better control of the pulmonary infection (9).

The purpose of the present research was to investigate the therapeutic effectiveness of bronchoalveolar lavage (BAL) under fiberoptic bronchoscopy in patients with severe pulmonary infection residing in the Tibetan plateau area, and to provide a clinical reference. We present the following article in accordance with the CONSORT reporting checklist (available at http://dx.doi.org/10.21037/ apm-21-470).

\section{Methods}

\section{Research participants}

This study enrolled 148 patients with severe pneumonia of various causes who were admitted to the comprehensive intensive care unit of Shigatse People's Hospital (Shigatse, Tibet Autonomous Region, China) between July 2019 and January 2021. Patients were randomly assigned to the observational group or the control group. The criteria for patient inclusion were: (I) severe lung infection confirmed by laboratory examination and imaging examination; (II) bronchoscopy and treatment could be tolerated. Patients with mental illnesses were excluded, as were those with a tendency to severe bleeding or with blood coagulation mechanism disorders. All procedures in this study involving human participants were performed in accordance with the Declaration of Helsinki (as revised in 2013). Informed consent was taken from all the patients. The study protocol was approved by the ethics committee of Shanghai Ninth People's Hospital (No. 2021RSYLL001).

\section{Study methods}

Basic clinical data for all patients, including sex, age, body mass index (BMI), blood pressure, diabetes history, stroke history, presence or absence of chronic obstructive pulmonary disease, lung infection (gram-positive bacterial infection, gram-negative bacterial infection, fungal infection, acute lung abscess), surgical history, and postoperative inhalation injury were collected. 
Control group patients received conventional treatment, such as anti-infective treatment, mechanical ventilation, and symptomatic treatment. In addition to the treatments received by the control group, patients in the observational group underwent BAL under fiberoptic bronchoscopy. Assess the patient's recovery after 1 month of treatment.

The specific operating procedures for BAL under fiberoptic bronchoscopy were as follows. The patient was given a subcutaneous injection of atropine 15 minutes before the operation. If the stress felt by the patient was too much, $10 \mathrm{mg}$ diazepam would be injected 30 minutes before the operation. After that, the patient was anesthetized with lidocaine, delivered by inhalation. The trachea was then checked with a fiberoptic bronchoscope, the sputum was sucked, and a bacterial sputum culture was carried out. Following that, the fiberoptic bronchoscope front end was extended to the bronchial opening of the lung segment for lavage. The special catheter of the fiberoptic bronchoscope was inserted into the biopsy hole for lavage in stages. Sterile normal saline was used as the lavage fluid; the temperature of the saline was maintained at $37^{\circ} \mathrm{C}$, and the volume of saline for each lavage was $10-15 \mathrm{~mL}$, with the total volume being less than $200 \mathrm{~mL}$. For each lung segment, lavage was performed 2 to 3 times. If a blood clot or spittoon was found during the procedure, it was crushed with a biopsy clip and sucked out. Finally, the fiberoptic bronchoscope was inserted into subsegments for the injection of amikacin, metronidazole, and amoxicillin. The injections were delivered over 5 to 10 minutes, once a day, for 1 week. Both the observational and control groups received the same comprehensive care during their treatment.

\section{Statistical analysis}

All data were expressed as the mean \pm standard deviation or number of cases (percentage). Differences between groups were compared by $t$-test, and chi-square test was used to evaluate percentage differences. Pearson's correlation was used to analyze the correlations between bronchial lavage under fiberoptic bronchoscopy and the levels of inflammatory factors. Logistic regression was used to analyze the correlation between the bronchial lavage under fiberoptic bronchoscopy and the effectiveness of the treatment. Statistical analyses were performed with SAS version 9.4 software (North Carolina State University, United States, North Carolina), and differences were statistically significant when $\mathrm{P}<0.05$.

\section{Results}

\section{Basic information of the research participants}

Of the 79 patients in the observational group, $58.2 \%$ were males. The average age was $52.4 \pm 5.3$ years old, and the average BMI was $24.9 \pm 3.4 \mathrm{~kg} / \mathrm{m}^{2}$. The number of grampositive bacterial, gram-negative bacterial, and fungal infections were 19 (24.1\%), 49 (62.0\%), and 11 (13.9\%), respectively. Thirty $(38.0 \%)$ and $19(24.1 \%)$ patients in the observational group had diabetes and chronic obstructive pulmonary disease, respectively, and $30(38.0 \%)$ patients had a history of surgery. Postoperative inhalation injury was reported in 17 (21.5\%) patients.

Comparison of the basic patient information of the observational and control groups showed that the 2 groups were similar before treatment, and there were no statistically significant differences (Table 1).

\section{Comparison of parameters of respiratory mechanics between the observational group and the control group after treatment}

As shown in Table 2, a comparative analysis of the respiratory mechanics parameters (chest compliance, lung compliance, and total dynamic compliance) of the patients in the observational group and the control group before treatment showed that there was no significant difference between the groups with respect to chest compliance, lung compliance, and total dynamic compliance (all $\mathrm{P}>0.05$ ). After treatment, the respiratory mechanics parameters in the 2 groups showed significant improvements compared with those before treatment (all $\mathrm{P}<0.05$ ). Furthermore, the parameters in the observational group were significantly higher compared to the control group (chest compliance: $73.81 \pm 8.42$ vs. $70.02 \pm 7.93 \mathrm{~mL} / \mathrm{cmH}_{2} \mathrm{O}$; lung compliance: $42.64 \pm 5.47$ vs. $35.88 \pm 5.31 \mathrm{~mL} / \mathrm{cmH}_{2} \mathrm{O}$; total dynamic compliance: $42.41 \pm 4.28$ vs. $33.76 \pm 3.09 \mathrm{~mL} / \mathrm{cmH}_{2} \mathrm{O}$ ) $(\mathrm{P}<0.05)$.

\section{Comparison of inflammatory factor levels between the observational group and control group after treatment}

As shown in Table 3, a comparative analysis of the inflammatory factor levels (PCT and TGF- $\beta$ ) of the observational group and the control group before treatment showed no statistically significant difference in the levels of PCT or TGF- $\beta$ (both $\mathrm{P}>0.05$ ). After 
Table 1 Comparison of basic data between the control group and the observational group

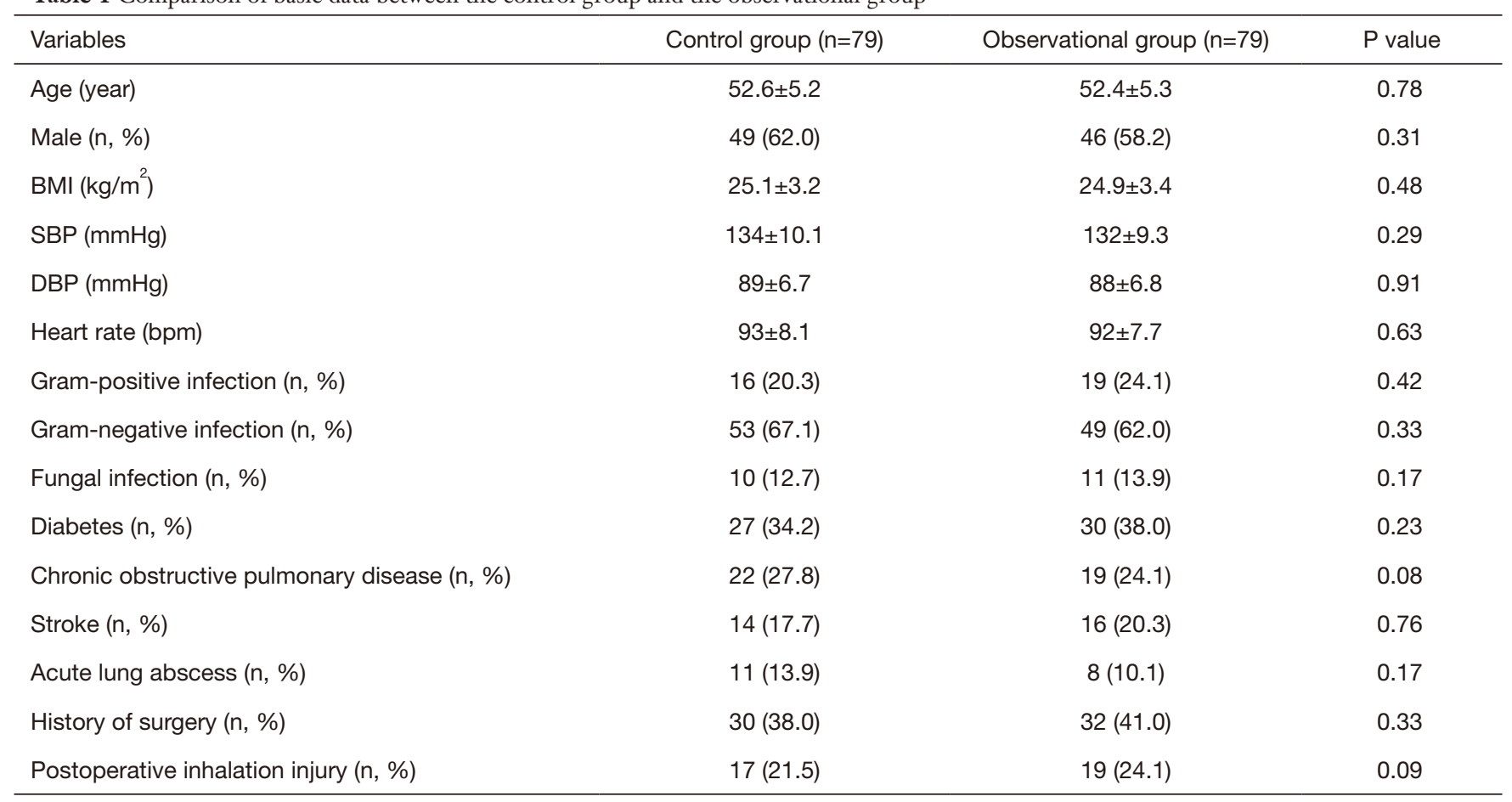

$\mathrm{n}$, the number of cases; BMI, body mass index; SBP, systolic blood pressure; DBP, diastolic blood pressure.

Table 2 Comparison of respiratory mechanics parameters between the control group and the observational group

\begin{tabular}{lcc}
\hline $\begin{array}{l}\text { Respiratory mechanics } \\
\text { parameters }\end{array}$ & $\begin{array}{c}\text { Observational } \\
\text { group }\end{array}$ & $\begin{array}{c}\text { Control } \\
\text { group }\end{array}$ \\
\hline
\end{tabular}

\begin{tabular}{ccc}
\hline Chest compliance $\left(\mathrm{mL} / \mathrm{cm} \mathrm{H}_{2} \mathrm{O}\right)$ & \\
Pre treatment & $55.32 \pm 7.42$ & $56.62 \pm 6.46$ \\
Post treatment & $73.81 \pm 8.42^{\star *}$ & $70.02 \pm 7.93^{\star}$
\end{tabular}

Lung compliance $\left(\mathrm{mL} / \mathrm{cm} \mathrm{H} \mathrm{H}_{2} \mathrm{O}\right)$

$\begin{array}{lll}\text { Pre treatment } & 36.68 \pm 5.52 & 30.92 \pm 5.02 \\ \text { Post treatment } & 44.71 \pm 5.47^{\star \#} & 35.88 \pm 5.31^{*}\end{array}$

Total dynamic compliance $\left(\mathrm{mL} / \mathrm{cm} \mathrm{H}_{2} \mathrm{O}\right)$

\begin{tabular}{lcc} 
Pre treatment & $26.16 \pm 2.42$ & $25.86 \pm 2.37$ \\
Post treatment & $42.41 \pm 4.28^{\star \#}$ & $33.76 \pm 3.09^{*}$ \\
\hline *, P $<0.05$ compared with before treatment; ${ }^{\#}, \mathrm{P}<0.05$ compared \\
with the control group.
\end{tabular}

treatment, the inflammatory factor indexes in both groups showed significant improvements compared with those before treatment (all $\mathrm{P}<0.05$ ). Furthermore, the levels of inflammatory factors in the observational group were significantly lower than those in the control group (PCT:
Table 3 Comparison of inflammatory factor indexes between the patients in the control group and observational group

\begin{tabular}{lcc}
\hline $\begin{array}{l}\text { Inflammatory } \\
\text { factors }\end{array}$ & $\begin{array}{c}\text { Observational } \\
\text { group }\end{array}$ & $\begin{array}{c}\text { Control } \\
\text { group }\end{array}$ \\
\hline PCT $(\mu \mathrm{g} / \mathrm{L})$ & $21.22 \pm 2.37$ & $19.69 \pm 2.41$ \\
Pre treatment & $2.51 \pm 0.47^{\star *}$ & $6.63 \pm 0.52$ \\
Post treatment & & \\
TGF- $\beta(\mathrm{ng} / \mathrm{L})$ & $114.55 \pm 10.78$ & $111.37 \pm 12.52$ \\
Pre treatment & $85.69 \pm 11.63^{\star \#}$ & $98.77 \pm 11.39$ \\
Post treatment &
\end{tabular}

*, $\mathrm{P}<0.05$ compared with the before treatment;, $\mathrm{P}<0.05$ compared with the control group. PCT, procalcitonin; TGF- $\beta$, transforming growth factor $\beta$.

$2.51 \pm 0.47$ vs. $6.63 \pm 0.52 \mu \mathrm{g} / \mathrm{L}$; TGF- $\beta$ : $85.69 \pm 11.63$ vs. $98.77 \pm 11.39 \mathrm{ng} / \mathrm{L})($ all $\mathrm{P}<0.05)$.

\section{Comparison of treatment effectiveness between the observational group and the control group}

As shown in Table 4, the results of the logistic regression 
Table 4 Comparison of treatment effectiveness in the observational group and control group

\begin{tabular}{lccc}
\hline Models & OR & $95 \% \mathrm{Cl}$ & P value \\
\hline Model 1 & 1.54 & $1.15-1.68$ & $<0.0001$ \\
Model 2 & 1.46 & $1.19-1.60$ & 0.0009 \\
Model 3 & 1.23 & $1.09-1.51$ & 0.0070 \\
\hline
\end{tabular}

OR, odds ratio; $\mathrm{Cl}$, confidence interval.

analysis showed that compared with the control group, without correction (model 1), the probability of the treatment in the observational group being effective was increased by $54 \%(\mathrm{OR}=1.54,95 \% \mathrm{CI}: 1.15-1.68, \mathrm{P}<0.0001)$. Compared with the control group, following adjustment for age and sex (model 2), the treatment effectiveness in the observational group was increased by $46 \%$ (OR $=1.46,95 \%$ CI: $1.19-1.60, \mathrm{P}=0.0009)$. Compared with the control group, finally, when BMI, gram-negative infection, diabetes, and acute lung abscess were adjusted (model 3), the treatment effectiveness in the observational group was increased by $23 \%(\mathrm{OR}=1.23,95 \% \mathrm{CI}: 1.09-1.51, \mathrm{P}=0.007)$.

\section{Discussion}

This study showed that, under similar clinical conditions, BAL under fiberoptic bronchoscopy significantly improves chest compliance, lung compliance, and total dynamic compliance, and decreases the levels of inflammatory factors (PCT and TGF- $\beta$ ), compared with conventional treatment, thus increasing the effectiveness of treatment.

Patients with severe pulmonary infections have limited systemic activity and reduced ability to excrete sputum independently, which can result in thick sputum and the accumulation of secretions, and thus increases the risk of airway blockage $(10,11)$. Studies have shown that the treatment of severe pulmonary infections should focus on fighting the infection, removing foreign bodies in the respiratory tract, and ensuring that the respiratory tract is clear of blockages (12). Studies have also found that current anti-infective treatments for severe pulmonary infections are not ideal. Recently, BAL under fiberoptic bronchoscopy has been gradually applied in clinical practice. Clinical experience has proved that fiberoptic bronchoscopy not only has the benefits of non-invasiveness and high efficacy, but that it can also reduce the impairment to airway mucosa $(13,14)$. Research has shown that small-cavity fiber bronchoscopes can penetrate small lesions, enable accurate observation of lung lesions directly, and provide dynamic and clear bronchial images $(15,16)$. These benefits are conducive to gaining an effective understanding of the progression of the disease, and to the clearing of respiratory secretions as well as foreign bodies, sputum, and blood clots, which can also help to alleviate bronchial obstructions in asthma and quickly enhance ventilation $(17,18)$. A European study of 400 patients with severe lung infections showed that BAL has a significant effect on improving lung infections (19). Our research demonstrated that BAL under fiberoptic bronchoscopy shortened the duration of mechanical ventilation and reduced the use of antibiotics compared with the control treatment. Furthermore, the effects of the treatment with respect to respiratory failure correction, lowering body temperature, and recovering white blood cells were also significantly better in the observation than in the control group. Therefore, our findings suggest that BAL under fiberoptic bronchoscopy can effectively relieve heavy bleeding, small airway interference, restore breathing, increase the oxygen content in the blood, and help to reduce cerebral edema, thereby accelerate the recovery. At the same time, the effectiveness of treatment in the observational group was significantly compared to the control group, suggesting that BAL can effectively improve the treatment effectiveness, which is consistent with the conclusions of previous studies (20).

The pulmonary infection of severity may affect alveolar oxygenation because of airway hinderance, which can lead to a disorder of oxygen and carbon dioxide exchange as well as changes in the patient's respiratory mechanics parameters (21). The results of this study showed that the respiratory mechanics parameters of patients following BAL under fiberoptic bronchoscopy were significantly improved compared to those of conventionally treated patients, and the treatment also showed significantly greater effectiveness than conventional treatment. These findings suggest that BAL under fiberoptic bronchoscopy can improve the respiratory status of patients.

Severe pulmonary infections have been reported to induce systemic inflammation and cause the unrestrained release of pro-inflammatory factors (22). PCT consists of calcitonin and $\mathrm{N}$-residues, which can inhibit the expression of the calcitonin gene when the host is not infected; therefore, in healthy people, the concentration of PCT is typically very low (23). However, during pathogenic infection, the expression of the calcitonin gene in the human body is up-regulated, which subsequently increases the level of PCT in the serum. Serum PCT can be used as a dependable index of severe infection to reveal the body's 
condition. TGF- $\beta$ is a polypeptide growth factor which is enriched in human kidney and lung tissues. It is typically released by lymphocytes and eosinophils, or by airway epithelial cells in the lungs (24). A variety of external stimuli can cause inflammation of the airways, thereby damaging the airway epithelium and increasing inflammation, as well as the secretion of TGF- $\beta$ (25). In this study, the patients in both the observational and control groups had reduced levels of PCT and TGF- $\beta$ postoperatively; however, the decrease in the observational group was more significant than that in the control group, which indicates that BAL under fiberoptic bronchoscopy can effectively improve the local microenvironment. Furthermore, when sensitive antibiotics were injected directly into the lesion under fiberoptic bronchoscopy, there was a higher concentration of antibiotics in the lesion than there was with intravenous delivery; thus, the former approach is more effective for achieving an anti-inflammatory effect than the latter. This study has certain limitations. First of all, the sample size of this study is small, and the conclusions of this study need to be verified in a larger study. Secondly, this study only included changes in PCT and TGF- $\beta$, and more inflammatory factors, such as IL-1 $\beta$, IL-6, etc., need to be included for a more adequate assessment of changes in inflammation.

In summary, the application of BAL under fiberoptic bronchoscopy for the treatment of severe pulmonary infections can effectively alleviate symptoms, improve the respiratory mechanics parameters, enhance the clinical efficacy, reduce complications, and reduce the levels of PCT and TGF- $\beta$. Therefore, BAL under fiberoptic bronchoscopy is worthy of wider clinical application. However, BAL under fiberoptic bronchoscopy is an invasive treatment, which has a certain invasion risk, and carries the risk of complications such as hypoxemia and hemopneumothorax. In clinical practice, adequate anesthesia and intraoperative monitoring should be used to avoid adverse events as much as possible. Although none of the patients in the current research experienced these complications, this may have resulted from the limited sample size in the current study. Thus, the safety of the clinical application of BAL under fiberoptic bronchoscopy needs to be ascertained through the performance of large-scale, multi-center studies in the future.

\section{Acknowledgments}

Funding: This study was supported by Shanghai Science and Technology Commission Fund (20035800300).

\section{Footnote}

Reporting Checklist: The authors have completed the CONSORT reporting checklist. Available at http://dx.doi. org/10.21037/apm-21-470

Data Sharing Statement: Available at http://dx.doi. org/10.21037/apm-21-470

Conflicts of Interest: All authors have completed the ICMJE uniform disclosure form (available at http://dx.doi. org/10.21037/apm-21-470). The authors have no conflicts of interest to declare.

Ethical Statement: The authors are accountable for all aspects of the work in ensuring that questions related to the accuracy or integrity of any part of the work are appropriately investigated and resolved. All procedures in this study involving human participants were performed in accordance with the Declaration of Helsinki (as revised in 2013). Informed consent was taken from all the patients. The study protocol was approved by the ethics committee of Shanghai Ninth People's Hospital (No. 2021RSYLL001).

Open Access Statement: This is an Open Access article distributed in accordance with the Creative Commons Attribution-NonCommercial-NoDerivs 4.0 International License (CC BY-NC-ND 4.0), which permits the noncommercial replication and distribution of the article with the strict proviso that no changes or edits are made and the original work is properly cited (including links to both the formal publication through the relevant DOI and the license). See: https://creativecommons.org/licenses/by-nc-nd/4.0/.

\section{References}

1. Kitami A, Kuroda Y, Ohashi S. Lung Cancer with Severe Infection Complicating Transbronchial Biopsy: Can This Complication Be Prevented? J Jpn Soc Resp Endosc 2016;38:476-84.

2. Tong S, Fan K, Jiang K, et al. Increased risk of severe infections in non-small-cell lung cancer patients treated with pemetrexed: A meta-analysis of randomized controlled trials. Curr Med Res Opin.2017;33:31-7.

3. Murat Sedef A, Kose F, Taner Sumbul A, et al. Prognostic value of procalcitonin infection-related mortality of cancer patients. J BUON 2016;21:740-4.

4. Ding FM, Zhu SL, Shen C, et al. Regulatory T cell activity is partly inhibited in a mouse model of chronic 
Pseudomonas aeruginosa lung infection. Exp Lung Res 2015;41:44-55.

5. Michiels A, Arsenakis I, Boyen F, et al. Efficacy of one dose vaccination against experimental infection with two Mycoplasma hyopneumoniae strains. BMC Vet Res 2017;13:274.

6. Xu ZB. Observation of efficacy of Xuebijing injection in combination with antibiotics in the treatment of ICU severe pulmonary Infection. Mod J Integr Chin Trad West Med 2016;7:431-2.

7. Liu W, Pang G, Wang S, et al. Protective effect of ulinastatin on severe pulmonary infection under immunosuppression and its molecular mechanism. Exp Ther Med 2017;14:3583-8.

8. Komiya K, Inagawa G, Nakamura K, et al. A simple fibreoptic assisted laryngoscope for paediatric difficult intubation: a manikin study. Anaesthesia 2009;64:425-9.

9. Soper TD, Haynor DR, Glenny RW, et al. In vivo validation of a hybrid tracking system for navigation of an ultrathin bronchoscope within peripheral airways. IEEE Trans Biomed Eng 2010;57:736-45.

10. Li L, Tan A, Chow V. Targeting vascular leakage for novel biomarker diagnosis and therapy of severe pulmonary infections. Int J Infect Dis 2018;73:118-9.

11. Liu Y, Sun JK, Qi X, et al. Expression and significance of Th17 and Treg cells in pulmonary infections with gramnegative bacteria. Immunol Invest 2017;46:730-41.

12. Hong G, Kim DH, Kim YS. Successful treatment of acute respiratory failure in a patient with pulmonary Mycobacterium abscessus infection accompanied by organizing pneumonia. J Thorac Dis 2017;9:E560-4.

13. Sun J, Ren BC, Yang F, et al. Effect of sputum suction by bronchofiberoscope combined with routine antiinfective therapy on lung function and inflammatory state in patients with severe lung infection. J HainanMed Coll 2016;22:1373-6.

14. Qamar W, Al-Ghadeer AR, Ali R, et al. Analysis of Trace Elements in Rat Bronchoalveolar Lavage Fluid by Inductively Coupled Plasma Mass Spectrometry. Biol Trace Elem Res 2017;178:246-52.

15. Efrati O, Gonik U, Bielorai B, et al. Fiberoptic bronchoscopy and bronchoalveolar lavage for the evaluation of pulmonary disease in children with primary immunodeficiency and cancer. Pediatr Blood Cancer 2007;48:324-9.

16. Maekawa K, Naka M, Shuto S, et al. The characteristics of patients with pulmonary Mycobacterium aviumintracellulare complex disease diagnosed by bronchial lavage culture compared to those diagnosed by sputum culture. J Infect Chemother 2017;23:604-8.

17. Chen G, Xu CQ, Huang XQ. Curative effect of bronchoalveolar lavage for pulmonary infection of patients undergoing mechanical ventilation. Chin JNosocomiol 2016;26:312-313, 328.

18. Chen SW, Wang TB, Tian YH, et al. Influence of fiberoptic bronchoscopy sputum suction on respiratory state and inflammatory stress of patients with severe pulmonary infection. Chin J Nosocomiol 2016;26:5122-4.

19. Dixon G, Lama-Lopez A, Bintcliffe OJ, et al. The role of serum procalcitonin in establishing the diagnosis and prognosis of pleural infection. Respir Res 2017;18:30.

20. Koutroulis I, Loscalzo SM, Kratimenos P, et al. Clinical Applications of Procalcitonin in Pediatrics: An Advanced Biomarker for Inflammation and Infection-Can It Also Be Used in Trauma? Int Sch Res Notices 2014;2014:286493.

21. Zhu Y. Tumor necrosis factor- $\alpha$ and procalcitonin level variations in the serum and their effects on organ function in patients with severe acute pancreatitis during infected stage. Pak J Pharm Sci 2017;30:1413-6.

22. Thomas BJ, Kan OK, Loveland KL, et al. In the shadow of fibrosis: innate immune suppression mediated by transforming growth factor- $\beta$. Am J Respir Cell Mol Biol 2016;55:759-66.

23. Rotolo MC, Pellegrini M, Martucci P, et al. Cannabinoids determination in bronchoalveolar lavages of cannabis smokers with lung disease. Clin Chem Lab Med 2019;57:498-503.

24. Reboul E. Vitamin E Bioavailability: Mechanisms of Intestinal Absorption in the Spotlight. Antioxidants (Basel) 2017;6:95.

25. Taylor J, Wiens T, Peterson J, et al. Characteristics of E-cigarette, or Vaping, Products Used by Patients with Associated Lung Injury and Products Seized by Law Enforcement - Minnesota, 2018 and 2019. MMWR Morb Mortal Wkly Rep 2019;68:1096-100.

English Language Editor: J. Reynolds

Cite this article as: Zhou W, Zhou C, Liu X, Shi N, Quyang W, Tu D, Xin Y, Ji L. A randomised trial on the therapeutic effectiveness of bronchoalveolar lavage under fiberoptic bronchoscopy in patients with severe lung infection living in the Tibetan plateau area. Ann Palliat Med 2021;10(3):3336-3342. doi: 10.21037/apm-21-470 